\title{
Axial CID and High Pressure Resonance CID in a Miniature Ion Trap Mass Spectrometer Using a Discontinuous Atmospheric Pressure Interface
}

\author{
Liang Gao, ${ }^{a}$ Guangtao Li, ${ }^{a}$ and R. Graham Cooks ${ }^{\mathrm{a}, \mathrm{b}}$ \\ ${ }^{a}$ Department of Chemistry, Purdue University, West Lafayette, Indiana, USA \\ b Analytical Instrumentation Development, Purdue University, West Lafayette, Indiana, USA
}

\begin{abstract}
Axial collision induced dissociation (CID) and high-pressure resonance CID were implemented and compared with normal low-pressure resonance CID in a miniature ion trap mass spectrometer to obtain more complete fragmentation spectra. Axial CID was realized simply by applying a potential to the discontinuous atmospheric pressure interface (DAPI) capillary without performing parent ion isolation before dissociation. High-pressure resonance CID employed a double-introduction pulse scan function, by means of which precursor ions isolated at low-pressure $\left(<10^{-3}\right.$ torr) were dissociated at high-pressure ( 0.1 torr- 1 torr) with higher excitation energy, so that tandem MS of isolated precursor ions was achieved and extensive fragmentation was obtained. A simple peptide (Leu-enkephalin) and dye molecule (rhodamine B) ionized by ESI were used to investigate both methods and compare them with normal low-pressure resonance CID. (J Am Soc Mass Spectrom 2010, 21, 209-214) ( 2010 American Society for Mass Spectrometry
\end{abstract}

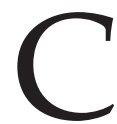
ollision-induced dissociation (CID) is often used to fragment mass-selected parent ions in tandem mass spectrometry [1-3]. Dissociation is achieved by energetic collisions with neutral gas molecules, resulting in internal energy accumulation and bond cleavage. Partial or complete precursor ion structure can be determined from the fragmentation information. The kinetic energy of the parent ions determines the amount of energy which can be converted to the internal energy and the collision frequency determines the conversion rate. Different kinetic energy deposition methods and collision pressure regimes usually give different fragmentation patterns of the precursor ions [4].

Resonance CID conducted at low-pressure $\left(<10^{-3}\right.$ torr) is commonly used to dissociate precursor ions in ion trap mass spectrometers $[5,6]$, and it is the usual way to dissociate ions in many miniature ion trap mass spectrometers [7-9]. In low-pressure resonance CID, kinetic energy is deposited by resonance excitation using an AC waveform set at the secular frequency of the precursor ions to be dissociated. The amplitude of the excitation AC waveform is usually small and the excitation period is usually long to achieve dissociation while still preventing the ejection of isolated ions [10, 11]. Because fragment ions are off-resonance with respect to the excitation signal, only a small amount of internal energy can be deposited and little sequential

Address reprint requests to Dr. R. Graham Cooks, Department of Chemistry, Purdue University, 560 Oval Drive, 1393 HC Brown Laboratory, West Lafayette, IN 47907-3084, USA. E-mail: cooks@purdue.edu fragmentation is obtained. Thus, low-pressure resonance CID is good at identifying specific low-energy fragmentation pathways. More complete fragmentation spectra usually can be obtained by axial CID or highpressure resonance CID compared with low-pressure resonance CID. Axial CID is usually realized by accelerating the primary ions in the axial direction of the collision cell, which is often a quadrupole operated at elevated pressure [12-14]. When the kinetic energy of the precursor ion is sufficiently high and collisions with background gas are frequent enough, the precursor ion internal energy increases rapidly above the dissociation threshold. If the precursor ions fragment fast in the region with a non-zero acceleration field, the fragment ions can be further accelerated and dissociated, resulting in sequential CID. A related method, "in-source CID", shares a similar fundamental mechanism with axial CID [15-17]. However the primary ions are not isolated and the dissociation is usually conducted in the skimmer region after the mass spectrometer orifice. Approaches to high-pressure resonance CID were reported on bench top mass spectrometers to achieve more efficient dissociation. A pulsed valve has been used in some benchtop mass spectrometers to introduce a gas pulse to increase the dissociation pressure (up to $10^{-3}$ torr $-10^{-2}$ torr) before implementing CID [18, 19], and an extra high-pressure ion trap $\left(10^{-3}\right.$ torr $-10^{-2}$ torr $)$ has been incorporated into other mass spectrometers to enhance dissociation [20].

The discontinuous atmospheric pressure interface (DAPI) was initially developed to couple atmospheric pressure ionization (API) sources to miniature ion trap 
mass spectrometers with limited pumping capabilities $[9,21,22]$. Using DAPI, gases carrying ions from an external ion source are pulsed into the ion trap at a high flow rate for short periods rather than continuously at a low flow rate. This allows API sources to be used with minimum requirements on the pumping system. During ion introduction through DAPI, the introduced ions undergo very frequent collisions with gas molecules due to the high-pressure ( $\sim 1$ torr). Therefore, dissociation can be achieved simply by providing sufficient kinetic energy to these ions.

In this communication, axial CID and high-pressure resonance CID are reported as means of fragmenting ions in miniature ion trap mass spectrometers. Axial CID was realized simply by applying a voltage to the DAPI capillary to create an accelerating electrical field at the exit of the DAPI; fragmentation spectra showing extensive dissociation were obtained. A high-pressure resonance CID method was also developed to isolate introduced ions at low-pressure and dissociate them at higher pressure, so that tandem MS with precursor ion isolation was achieved compared to axial CID and fragmentation as extensive as that of axial CID can be obtained at the same time.

\section{Experimental}

A modified Mini 10 mass spectrometer with DAPI was used in these experiments (a differential rf rather than a single phase rf was used to drive the RIT). The configuration of the mass analyzer assembly is shown in Figure 1. During operation, ions generated at atmosphere are transferred via DAPI into a standard rectilinear ion trap, driven by a $1 \mathrm{MHz}$ differential rf signal without DC offset, for mass analysis [23]. An electron multiplier (Detech 2312; Detector Technology Inc., Palmer, MA, USA) is used to detect ions ejected from the ion trap. The DAPI interface consists of two stainless steel capillaries S1 (i.d. $0.25 \mathrm{~mm}$, o.d. $1.59 \mathrm{~mm}$, length $100 \mathrm{~mm}$ ) and S2 (i.d. $1.2 \mathrm{~mm}$, o.d. $1.59 \mathrm{~mm}$, length 50 $\mathrm{mm})$, connected to each other via a conductive silicone tube (i.d. $1.59 \mathrm{~mm}$, o.d. $3.18 \mathrm{~mm}$, length $5 \mathrm{~cm}$; Simolex Rubber Corp., Plymouth, MI, USA). The conductive silicone tube goes through a normally closed pinch valve (390NC24330, ASCO Valve Inc., Florham Park, NJ), which controls the open/close status of the interface. An adjust-

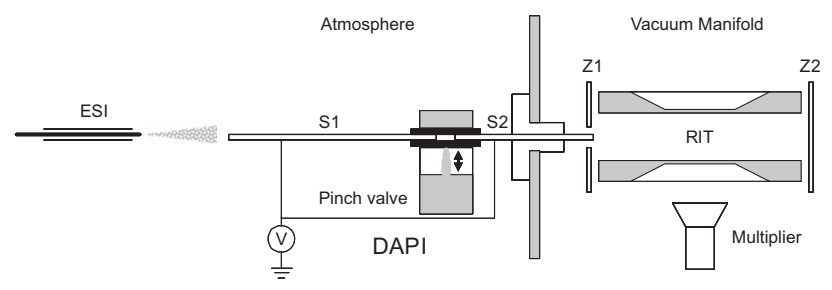

Figure 1. Configuration of the mass analyzer, including discontinuous interface (DAPI) connecting the atmospheric pressure region to the vacuum region, rectilinear ion trap, and electron multiplier. able potential, rather than ground as in previous studies, is applied to the capillaries S1 and S2.

In the standard scan function, a $+24 \mathrm{~V}$ DC pulse signal lasting for a few milliseconds is applied to the pinch valve to open the interface for ion introduction; then the interface is closed and the pumping system reduces the manifold pressure to its base value in a period ranging from several hundred milliseconds to a few seconds. Thereafter, the multiplier is turned on; both the rf signal amplitude and resonance AC signal amplitude are scanned from low to high values to eject ions in mass sequence from the ion trap and also to record a mass spectrum. The DC voltages on both ion trap z-endcap electrodes are maintained at $+20 \mathrm{~V}$ during the whole scan cycle. The scan function may vary a little for different purposes, as given for specific cases later.

An external electrospray ionization (ESI) source operated at $+4 \mathrm{kV}$ DC voltage and 80 psi sheath gas pressure applied was used as the ionization source in all experiments. The electrospray sprayer was made using a Swagelok T-piece and two coaxial capillary tubing. The internal fused silica capillary had an i.d. of $100 \mu \mathrm{m}$ and an o.d. of $190 \mu \mathrm{m}$. It extended through the T-piece and was connected to a syringe pump. The outer silica capillary tubing had an i.d. of $250 \mu \mathrm{m}$ and an o.d. of $0.5 \mathrm{~mm}$ and was $10 \mathrm{~mm}$ long. All samples were prepared with 1:1 methanol/water solution with $1 \%$ acetic acid and supplied at a flow rate of $1 \mu \mathrm{L} / \mathrm{min}$ during analysis.

\section{Results and Discussion}

A 5 ppm Leu-enkephalin (YGGFL) peptide solution was ionized by ESI and used to demonstrate axial CID. Leu-enkephalin was selected because the relationship between its fragmentation pattern and internal energy is well understood. A scan function with $15 \mathrm{~ms}$ ion introduction time and $1 \mathrm{~s}$ pumping time was used in this experiment. Mass spectra were recorded at DAPI capillary voltages varying from 0 to $+220 \mathrm{~V}$ at $10 \mathrm{~V}$ intervals, while the voltages on both z-endcap electrodes of the ion trap were maintained at $+20 \mathrm{~V}$. Typical mass spectra collected at voltages of $0,+70$, $+90,+100,+110$, and $+130 \mathrm{~V}$ are shown in Figure $2 \mathrm{a}$. The intensity of protonated YGGFL as well as major fragment ions $b_{4}, a_{4}, b_{3} \& y_{2}$, and $a_{1}$ are plotted versus the capillary voltage from 0 to $+130 \mathrm{~V}$ in Figure $2 b\left(b_{3}\right.$ and $y_{2}$ are plotted together since they are not always well separated in the miniature mass spectrometer). The results show little fragmentation until the capillary voltage rises to $+70 \mathrm{~V}$, which confirms that the translational energy does not provide sufficient energy to dissociate the introduced ions. However, decreased baseline noise and increased precursor ion $(\mathrm{m} / \mathrm{z} 556)$ signal intensity (by just $45 \%$ when the DAPI capillary voltage increased from 0 to $+60 \mathrm{~V}$ ), which corresponds to a higher trapping efficiency, were observed with increasing capillary voltage. The improved signal to 

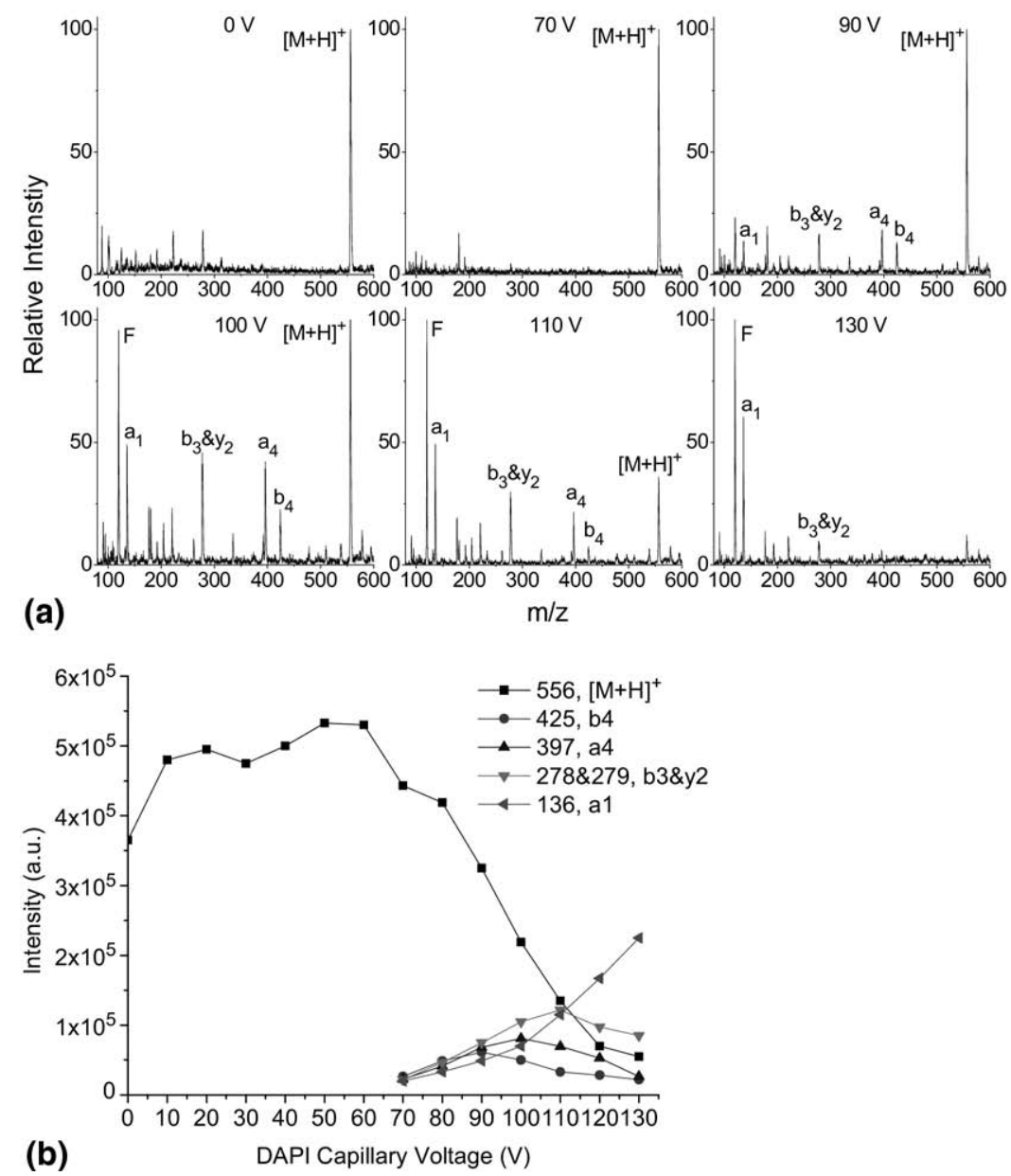

Figure 2. (a) Axial CID spectra of protonated YGGFL obtained by applying DAPI capillary voltages of $0,+70,+90,+100,+110$, and $+130 \mathrm{~V}$. (b) Intensity variation of protonated YGGFL and major fragment ions $\mathrm{b}_{4}, \mathrm{a}_{4}, \mathrm{~b}_{3}$ and $\mathrm{y}_{2}$, and $\mathrm{a}_{1}$ as a function of DAPI capillary voltages from +70 to $+130 \mathrm{~V}$.

noise ratio and increased intensity of the precursor ion are ascribed to the dissociation of background and matrix derived ions, e.g., solvent adducts, similar to observations reported for in-source CID [24, 25]. Fragment ions are observed in abundance when the DAPI capillary voltage reaches $+80 \mathrm{~V}$, and the intensity ratio of low $\mathrm{m} / \mathrm{z}$ and high $\mathrm{m} / \mathrm{z}$ fragment ions increases with continued increase in the voltage, indicating increased internal energy deposition and sequential CID. The appearance of the low $\mathrm{m} / \mathrm{z}$ fragment ions also suggests a rapid dissociation process, in which initial fragmentation of the original precursor ions occurs in the region close to the DAPI exit where the axial acceleration field is non-zero, and initially formed fragments are further accelerated and dissociated. No ions could be detected after the capillary voltage reached $+220 \mathrm{~V}$, showing that all ions are lost at a high DAPI capillary voltage. There are two explanations for this latter phenomenon. One is that although the acceleration field in mainly in the axial direction of the ion trap, there are still field components in the radial direction. Once the DAPI capillary voltage is large enough, this field causes the loss of introduced ions. Also, the kinetic energy of the introduced ions gained in the axial direction can be transferred in part to the radial direction through collisions which again causes loss of introduced ions. The other reason is that product ions may further dissociate until the product ion's mass to charge ratio is lower than the low mass cut off, which causes ion loss. It is hard to identify the major factor in the experiment, although the common result is that all ions are eventually lost. Besides the double-phase rf operation used in the RIT system presented, axial CID is also applicable to single-phase rf RIT systems although the experimental parameters are different since the center potential of a single-phase rf-driven RIT varies over a large range. The same experiment was repeated using a $5 \mathrm{ppm}$ rhodamine B solution, and the results are shown in the Supporting Information. Ions produced by multiple stages fragmentation were able to be obtained simply by raising the DAPI capillary voltage.

To compare the axial CID experiment described above with normal low-pressure resonance CID, the fragmentation spectrum of Leu-enkephalin was recorded using both methods. The axial CID spectrum shown in Figure 3a was obtained simply by applying 


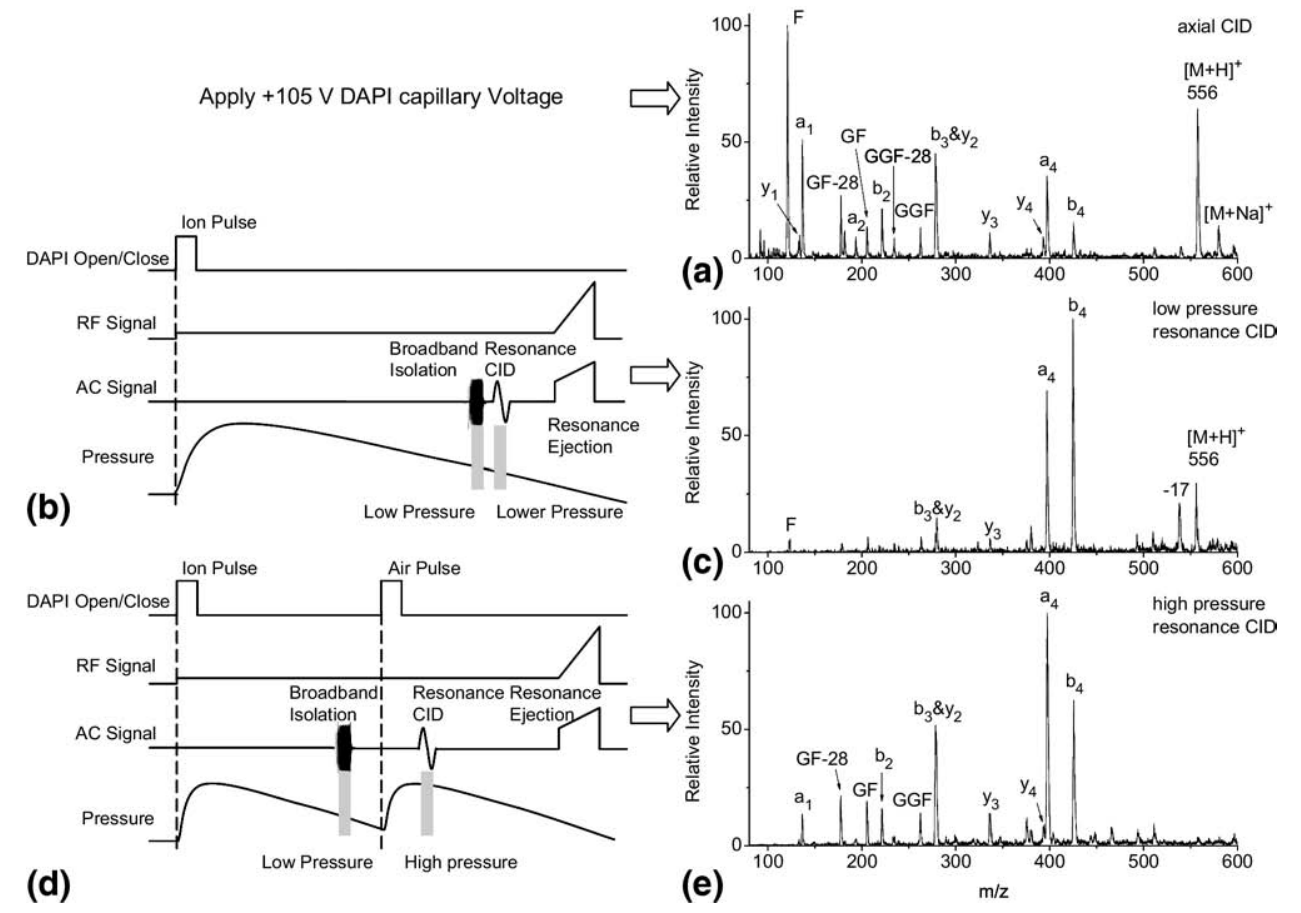

Figure 3. (a) Fragmentation spectra of protonated YGGFL obtained by axial CID at +105 V DAPI capillary voltage. (b) Scan function used for normal low-pressure resonance CID, precursor ions are isolated at low-pressure by broadband isolation and dissociate at lower pressure by resonance CID, dissociation time is long and excitation energy is small. (c) Fragmentation spectra of protonated YGGFL obtained by low-pressure resonance CID at +60 V DAPI capillary voltage. (d) Double-pulse scan function used for high-pressure resonance CID, parent ions are isolated after the first ion introduction at low-pressure, and dissociated after the second air introduction pulse at high-pressure by resonance CID, dissociation time is short and excitation energy is large. The actual length of the scan function is twice of that of the scan function used for low-pressure resonance CID. (e) Fragmentation spectra of protonated YGGFL obtained by high-pressure resonance CID at +60 V DAPI capillary voltage.

$+105 \mathrm{~V}$ DC to the DAPI capillary. The scan function shown in Figure $3 \mathrm{~b}$ was used to obtain the low-pressure resonance CID spectrum with a $+60 \mathrm{~V}$ DC voltage applied to the DAPI capillary. Protonated YGGFL molecules with $\mathrm{m} / \mathrm{z} 556$ were first isolated as precursor ions by applying a notched broadband waveform for $10 \mathrm{~ms}$, after a $750 \mathrm{~ms}$ pumping period. Then a $0.2 \mathrm{~V}_{p-p}, 67 \mathrm{kHz}$ resonance AC waveform $(q \approx 0.19)$ was applied for 50 ms immediately after the isolation to dissociate the isolated ions at a background pressure of $\sim 10^{-3}$ torr. The product ion spectrum shown in Figure $3 \mathrm{c}$ was finally obtained by resonance ejection of all fragment ions after another $200 \mathrm{~ms}$ pumping time. In the lowpressure resonance CID spectrum Figure $3 c$, fragment ions $b_{4}$ and $a_{4}$ are dominant ions, corresponding to the fragments from a major dissociation pathway of $\mathrm{P} \rightarrow \mathrm{b}_{4} \rightarrow \mathrm{a}_{4}$, which requires little internal energy [26]. On the other hand, many more fragment ions appear in the axial dissociation spectrum Figure 3a, which is similar to that obtained by axial CID in the quadrupole collision cell of a Q-TOF mass spectrometer [26]. The higher internal energy deposition is apparent.

The difference in dissociation pressures (1 torr and $10^{-3}$ torr, the pressure estimation is validated in the Supporting Information) of the two methods contrib- utes to the different fragmentation patterns. In axial CID, the high accelerating field causes dissociation of introduced ions though high-frequency collisions with gas molecules $\left(\sim 10^{7} \mathrm{~Hz}\right)$ rather than allowing their escape from the ion trap [27]. The excitation energy is applied in the axial direction, so that there is no limitation on the dissociation $q$ value of the parent ions $(q \approx 0.1$ in Figure 3a), which is another reason why a more complete product ion spectrum is obtained by axial CID. However, in low-pressure resonance CID, parent ions isolation must be conducted at low-pressure to achieve efficient isolation, so that the following CID event is conducted at even lower pressure. The excitation energy transfer must therefore be low, and the dissociation $q$ value of parent ions has to be reasonably high during the dissociation process, otherwise, the precursor ions will be ejected from the ion trap because of insufficient numbers of cooling collisions with neutral molecules. Both the advantages and the disadvantage of axial CID are therefore obvious, the latter following from the fact that no prior ion isolation can be performed before dissociation, making it unsuitable for complex mixture analysis.

To achieve both precursor ion isolation and also obtain extensive fragmentation of the precursor ion at 
the same time, a high-pressure resonance CID method was developed for miniature ion trap mass spectrometers fitted with DAPI using the double-pulse scan function shown in Figure 3d. In this method, precursor ion isolation is performed after the first ion introduction pulse as usual at low-pressure $\left(<10^{-3}\right.$ torr $)$. After that, the pinch valve is opened again and only air is introduced in the second pulse, then resonance CID is conducted. The dissociation pressure can be controlled easily by changing the air pulse width and the pumping time between the second air pulse and CID. Therefore, the isolated precursor ion can be dissociated at highpressure (0.1 torr-1 torr), allowing more excitation energy to be applied to achieve more complete fragmentation. (The Supporting Information shows the small loss in the isolated parent ions due to the air introduction pulse). The excitation resolution of highpressure resonance CID may drop due to the increased pressure, which is due to the uncertainty of the ion secular frequency. The result is that ions being excited can pick up less energy from the excitation field. However, this can be compensated for by increasing the excitation signal amplitude.

In these experiments, the DAPI capillary voltage was maintained at $+60 \mathrm{~V}$. Protonated YGGFL molecules generated by ESI were introduced into the ion trap in the first pulse lasting for $15 \mathrm{~ms}$ and a notched broadband waveform was applied to isolate precursor ions of $\mathrm{m} / \mathrm{z} 556$ after a sufficiently long pumping time (750 ms). Then, the pinch valve was opened again for another 10 $\mathrm{ms}$, the ESI source was blocked this time so that only air was introduced (air pulse introduction can also be achieved using another pinch valve). Next, a $1.5 \mathrm{~V}_{p-p}, 67$ $\mathrm{kHz}$ resonance AC waveform $(q \approx 0.19)$ lasting for $2 \mathrm{~ms}$ was applied to dissociated the isolated ions at highpressure ( 0.1 torr -1 torr) $80 \mathrm{~ms}$ after the air introduction pulse. After another $1 \mathrm{~s}$ pumping period, fragment ions were ejected and mass analyzed using resonance ejection. A fragmentation efficiency of $70 \%$ was obtained from the resulting mass spectrum shown in Figure 3e. It can be observed that the fragment ions obtained are very similar to those recorded using axial CID except that the intensity of low $\mathrm{m} / \mathrm{z}$ fragment ions is lower, mainly due to the higher dissociation $q$ value of the precursor ions. Note that the dissociation $q$ value in resonance CID has to be higher than that used in axial CID because the trapping potential well depth in the radial direction has to be high enough to prevent the escape of the precursor ions during resonance excitation, which is also in the radial direction. Another difference is that almost all precursor ions were dissociated due to the efficient resonance excitation. The high-pressure resonance CID obviously has the advantages of parent ion isolation and complete fragmentation of precursor ions. Also, the parent ions can be accumulated using multiple ion introduction pulses method for several cycles before the final dissociation step to achieve a better signal to noise ratio [22]. Nevertheless, axial CID still holds the advantages of being easily achievable for all ions, and of operating at low $q$ values of the precursor ions. Moreover, by dissociating all introduced ions simultaneously, axial CID may be used to identify several compounds of interest simultaneously by comparing the obtained mass spectrum with the fragmentation spectrum of each of them. Peptide GFSPFR was used as another example to compare these CID methods in the Supporting Information with analogous results.

\section{Conclusion}

Axial CID and high-pressure resonance CID were used in a miniature ion trap mass spectrometer operating with DAPI interface to obtain more complete fragmentation spectra than that available with convention low-pressure resonance CID. In axial CID, extensive fragmentation was obtained due to the higher dissociation pressure, higher excitation energy, and lower dissociation $q$ value of the precursor ions. This method is easily implemented for all ions, but there is no precursor ion isolation. High-pressure resonance CID using a doublepulse scan function was developed to achieve tandem MS with precursor ion isolation and more complete fragmentation. Precursor ions isolated at low-pressure are dissociated in high-pressure by resonance CID, so that higher CID dissociation energy can be applied compared with low-pressure resonance CID. Both new methods presented appear to have value in improving analytical capabilities of miniature ion trap mass spectrometers.

\section{Acknowledgments}

The authors acknowledge support for this work by NSF 0848650CHE. L.G. acknowledges a graduate fellowship from the ACS Division of Analytical Chemistry, sponsored by Agilent Technology Inc.

\section{Appendix A Supplementary Material}

Supplementary material associated with this article may be found in the online version at doi:10.1016/ j.jasms.2009.10.005.

\section{References}

1. Jennings, K. R. Collision-Induced Decompositions of Aromatic Molecular Ions. Int. J. Mass Spectrom. Ion Phys. 1968, 1, 227-235.

2. Kondrat, R. W.; Cooks, R. G. Direct Analysis of Mixtures by Mass Spectrometry. Anal. Chem. 1978, 50, 81A-92A.

3. McLafferty, F. W.; Bockhoff, F. M. Separation/Identification System for Complex Mixtures using Mass Separation and Mass Spectral Characterization. Anal. Chem. 1978, 50, 69-76.

4. Sleno, L.; Volmer, D. A. Ion Activation Methods for Tandem Mass Spectrometry. J. Mass Spectrom. 2004, 39, 1091-1112.

5. Louris, J. N.; Cooks, R. G.; Syka, J. E. P.; Kelley, P. E.; Stafford Jr., G. C.; Todd, J. F. J. Instrumentation, Applications, and Energy Deposition in Quadrupole Ion-Trap Tandem Mass Spectrometry. Anal. Chem. 1987, 59, 1677-1685.

6. March, R. E. An Introduction to Quadrupole Ion Trap Mass Spectrometry. J. Mass Spectrom. 1997, 32, 351-369. 
7. Gao, L.; Song, Q.; Patterson, G. E.; Cooks, R. G.; Ouyang, Z. Handheld Rectilinear Ion Trap Mass Spectrometer. Anal. Chem. 2006, 78, 59946002.

8. Contreras, J. A.; Murray, J. A.; Tolley, S. E.; Oliphant, J. L.; Tolley, H. D.; Lammert, S. A.; Lee, D. E.; Later, D. W.; Lee, M. L. Hand-Portable Gass Chromatograph-Toroidal Ion Trap Mass Spectrometer (GC-TMS) for Detection of Hazardous Compounds. J. Am. Soc. Mass Spectrom. 2008, $19,1425-1434$

9. Gao, L.; Sugiarto, A.; Haper, J. D.; Cooks, R. G.; Ouyang, Z. Design and Characterization of a Multisource Hand-Held Tandem Mass Spectrometer. Anal. Chem. 2008, 80, 7198-7205.

10. Charles, M. J.; McLuckey, S. A.; Glish, G. L. Competition between Resonance Ejection and Ion Dissociation during Resonant Excitation in a Quadrupole Ion Trap. J. Am. Soc. Mass Spectrom. 1994, 5, 1031-1041.

11. Collings, B. A. Fragmentation of Ions in a Low Pressure Linear Ion Trap. J. Am. Soc. Mass Spectrom. 2007, 18, 1459-1466.

12. Yost, R. A.; Enke, C. G. Selected Ion Fragmentation with a Tandem Quadrupole Mass Spectrometer. J. Am. Chem. Soc. 1978, 7, 2274-2275.

13. Yost, R. A.; Enke, C. G. High Efficiency Collision-Induced Dissociation in an rf-only Quadrupole. Int. J. Mass Spectrom. Ion Phys. 1979, 30, $127-136$

14. Hopfgartner, G.; Varesio, E.; Tschappat, V.; Grivet, C.; Bourgogne, E.; Leuthold, L. A. Triple Quadrupole Linear Ion Trap Mass Spectrometer for the Analysis of Small Molecules and Macromolecules. J. Mass Spectrom. 2004, 39, 845-855.

15. Kambara, H.; Kanomata, I. Determination of Impurities in Gases by Atmospheric Pressure Ionization Mass Spectrometry. Anal. Chem. 1977, $49,270-275$

16. Bruins, A. P. Mass Spectrometry with Ion Sources Operating at Atmospheric Pressure. Mass Spectrom. Rev. 1991, 10, 53-77.

17. Weinmann, W.; Stoertzel, M.; Vogt, S.; Svoboda, M.; Schreiber, A. Tuning Compounds for Electrospray Ionization/In-Source Collision- induced Dissociation and Mass Spectra Library Searching. J. Mass Spectrom. 2001, 36, 1013-1023.

18. Doroshenko, V. M.; Cotter, R. J. Pulsed Gas Introduction for Increasing Peptide CID Efficiency in a MALDI/Quadrupole Ion Trap Mass Spectrometer. Anal. Chem. 1996, 68, 463-472.

19. Collings, B. A.; Romaschin, M. A. MS/MS of Ions in a Low Pressure Linear Ion Trap using a Pulsed Gas. J. Am. Soc. Mass Spectrom. 2009, 20, 1714-1717.

20. Thermo Fisher Scientific Inc. Dual-Pressure Linear Ion Trap Technology.

21. Gao, L.; Cooks, R. G.; Ouyang, Z. Breaking the Pumping Speed Barrier in Mass Spectrometry: Discontinuous Atmospheric Pressure Interface. Anal. Chem. 2008, 80, 4026-4032.

22. Gao, L.; Li, G.; Nie, Z.; Duncan, J.; Ouyang, Z.; Cooks, R. G. Characterization of a Discontinuous Atmospheric Pressure Interface. Multiple Ion Introduction Pulses for Improved Performance. Int. J. Mass Spectrom. 2009, 283, 30-34.

23. Ouyang, Z: Wu, G.; Song, Y. Li, H. Plass, W. R.; Cooks, R. G. Rectilinear Ion Trap: Concepts, Calculations, and Analytical Performance of a New Mass Analyzer. Anal. Chem. 2004, 76, 4595-4605.

24. Kambara, H.; Kanomata, I. Collision-Induced Dissociation of Water Cluster Ions at High Pressure. Int. J. Mass Spectrom. Ion Phys. 1977, 25 , 129-136.

25. Ketkar, S. N.; Dulak, J. G.; Fite, W. L.; Buchner, J. D.; Dheandhanoo, S Atmospheric Pressure Ionization Tandem Mass Spectrometric System for Real-Time Detection of Low-Level Pollutants in Air. Anal. Chem. $1989,61,260-264$.

26. Rakov, V. S. Borisov, O. V.; Whitehouse, C. M. Establishing LowEnergy Sequential Decomposition Pathways of Leucine Enkephalin and Its N- and C-Terminus Fragments Using Multiple-Resonance CID in Quadrupolar Ion Guide. J. Am. Soc. Mass Spectrom. 2004, 15, 1794-1809.

27. Dushman, S. Scientific Foundations of Vacuum Technique, 2nd ed.; Wiley, New York, 1962. 